\title{
Ethylene signaling modulates contents of catechin and ability of antioxidant in Camellia sinensis
}

Shun-Wun Ke', Guan-Heng Chen², Chung-Tse Chen², Jason T. C. Tzen² and Chin-Ying Yang ${ }^{1 *}$ (D)

\begin{abstract}
Background: Tea is one of the most popular beverages in the world. There are many secondary metabolites can be found in tea such as anthocyanins, proanthocyanidins, flavonols and catechins. These secondary metabolites in plants are proved to act protective components for human health effect. Plant hormone ethylene is considered to have an important role in regulation of plant development and signal transduction. This study evaluated the effect of ethylene signaling regulation in phenolic compounds in tea plants. The ethylene precursor 1-aminocyclopropane-1-carboxylic acid (ACC) enhanced contents of total catechin in treated oolong tea seedlings.

Results: The degree of epigallocatechin and epicatechin galloylation was increased after ACC treatment in oolong tea seedlings by high performance liquid chromatography determination. The contents of anthocyanins, flavonoids, and total polyphenol were higher after ACC treatment in comparison with control. Antioxidant enzyme such as catalase, superoxide dismutase, and total peroxidase decreased their antioxidant activities after ACC treatment, yet the activity of ascorbate peroxidase is increased. The ability of oxygen radical absorption and 2,2-diphenyl-1-picrylhydrazyl was used to evaluate the antioxidant activity, which was enhanced by ACC treatment.
\end{abstract}

Conclusions: Taken together the results of this study demonstrate that the ethylene signaling is involved in modulation of secondary metabolites accumulation and antioxidant ability that to enhance the benefit of human health in tea products.

Keywords: Ethylene, Antioxidant, Catechins, Polyphenol, Tea plant

\section{Background}

Tea (Camellia sinensis L.) is an evergreen shrub and prevalent commercial crop grown in more than 50 countries. Tea plants are rich in secondary metabolites, which have potential health benefits for humans (Zhou et al. 2016). Secondary metabolic products in tea include flavonoids, polyphenols, theanine, and alkaloids. Flavonoids are a large group of phenolic secondary metabolites including anthocyanins, proanthocyanidins, phenolic acids, flavonols and catechins (Abeynayake et al. 2012; Sun et al. 2016). The predominant flavonoids in tea are

\footnotetext{
*Correspondence: emiyang@dragon.nchu.edu.tw

1 Department of Agronomy, National Chung Hsing University,

Taichung 40227, Taiwan

Full list of author information is available at the end of the article
}

catechins (flavan-3-ols), which are generally classified into the following groups such as epicatechin (EC), epicatechin-gallate (ECG), epigallocatechin (EGC) and epigallocatechin-gallate (EGCG) (Perva-Uzunalic et al. 2006).

The production of secondary metabolites in higher plants is often affected by hormone regulation. Ethylene is a plant hormone that affects the plants growth and development and is sometimes required for processes including ripening, senescence, and abscission (Schaller 2012). In tea plants, floral buds are more sensitive to ethylene than are leaves (Woolf et al. 1999). Ethylene is critical for signal transduction and its effect on the accumulation of secondary metabolites. Ethylene regulates flavonol biosynthesis through the MYB12 transcription factor, which affects the expression of chalcone synthase, 
chalcone isomerase, and flavonol synthase (Lewis et al. 2011). Ethylene signaling is involved in the defense resistance of plants by enhancing polyphenol oxidase activity during stress (Bosch et al. 2014; Thipyapong and Steffens 1997). Studies on rice coleoptiles have shown that ethylene induces the accumulation of polyamine (Lee and Chu 1992).

Previous studies have shown that ethylene signaling contributes to the accumulation of secondary metabolites to facilitate defense resistance in plants. However, little is known about ethylene signaling regulation in the phenolic compounds in tea plants. 1-aminocyclopropane1-carboxylic acid (ACC) is known to be a direct precursor of ethylene and is transported throughout the plant over short and long distances (Boller et al. 1979), it is believed that the responses observed in this study is caused by ethylene. Our results demonstrated that after treatment with the ethylene precursor ACC, the total catechin content in oolong tea seedlings increased. High-performance liquid chromatography (HPLC) indicated that the degrees of EGC and EC galloylation were increased by ACC treatment in seedlings of oolong tea. The accumulation of phenolic compounds was enhanced after ACC treatment such as anthocyanins, flavonoids, and total polyphenol. The activities of catalase (CAT), superoxide dismutase (SOD), and total peroxidase (POX) decreased and ascorbate peroxidase (APX) increased after ACC treatment by antioxidant enzyme activity assay. Ability of antioxidant was enhanced after ACC treatment via evaluated according to the oxygen radical absorption capacity (ORAC), and 2,2-diphenyl-1-picrylhydrazyl (DPPH). Our results demonstrate that the contents of secondary metabolites and capacity of antioxidant can be increased through modulating ethylene signaling pathways in tea.

\section{Methods}

\section{Plant materials and treatments}

Tea [Camellia sinensis (L.) Kuntze] cultivar Chin-Shin Oolong was used in the experiments in this study. All seedlings of the tea plant were grown in the same area in Nantou, Central Taiwan, over a 2-year period. The tea plant seedlings were approximately $50 \mathrm{~cm}$ high. For ACC treatment, 2-year-old tea seedlings were irrigated with $20 \mathrm{~mL}$ of $100 \mu \mathrm{M}$ ACC per day for 5 days; the control (CK) samples were irrigated with $20 \mathrm{~mL}$ of water instead. Fresh samples each with one tip and two leaves were collected from tea seedlings, frozen immediately in liquid nitrogen, and stored at $-80{ }^{\circ} \mathrm{C}$ until analysis.

\section{Preparation of extracts from young tea seedling leaves}

The samples were weighed, and lyophilized samples were homogenized to powder by using a disposable pestle. The extraction of tea was prepared with $1.0 \mathrm{~mL}$ of $75 \%$ ethanol and shaking the mixture vigorously with an Intelli-Mixer (MyLabTM RM-2 M) for $2 \mathrm{~h}$ at room temperature. The mixture extraction of tea was filtered by $0.45 \mu \mathrm{m}$ polyvinylidene difluoride membrane filter (Pall Corporation, Glen Cove, NY, USA) before HPLC analysis.

\section{HPLC analysis}

Tea extracts were analyzed on a liquid chromatography system coupled with a Model 600E photodiode array detector (Waters Corporation, Milford, MA, USA). The chromatographic separation was performed on a Mightysil RP-18 GP column $(250 \times 4.6 \mathrm{~mm}$ i.d., $5 \mu \mathrm{m}$; Kanto Chemical Co., Tokyo, Japan). The mobile phase consisted of water containing $0.5 \%$ acetic acid (solvent $\mathrm{A}$ ) and acetonitrile (solvent B).

The samples were eluted using the following gradient program, $0 \mathrm{~min}, 95 \% \mathrm{~A}$ and $5 \% \mathrm{~B} ; 100 \mathrm{~min}, 75 \% \mathrm{~A}$ and $25 \% \mathrm{~B}$. The column was kept at room temperature, the volume of each injection was $10 \mu \mathrm{L}$, and the flow rate was $1 \mathrm{~mL} / \mathrm{min}$. The PDA detector was set at $280 \mathrm{~nm}$. The four major catechins (EC, EGC, ECG, and EGCG) shown in the HPLC profiles were identified as described in a previous study (Chen et al. 2014).

\section{Determination of anthocyanins, flavonoids, and total phenol}

The tea seedling samples $(0.1 \mathrm{~g})$ were excised and immediately used for anthocyanin, flavonoid, and total phenol content assays. For the anthocyanin and flavonoid content assays, the sample extracts in $2 \mathrm{~mL}$ of potassium phosphate buffer $(100 \mathrm{mM}, \mathrm{pH} 7.8)$ were ground into powder with liquid nitrogen. The mixture of tea extraction was centrifuged at $16,000 \mathrm{~g}$ and $4{ }^{\circ} \mathrm{C}$ for $30 \mathrm{~min}$, and the resultant supernatant was measured at 600 and $320 \mathrm{~nm}$ by using a spectrophotometer (Metertec SP8001). One absorbance unit was defined as the quantity of anthocyanins and flavonoids that exhibits an absorbance of 1 at 600 and $320 \mathrm{~nm}$. The total phenolic content assay was performed using a modified form of the procedure described by Singleton and Rossi (1965). The sample extracts in $1.0 \mathrm{~mL}$ of $75 \%$ ethanol were ground into powder with liquid nitrogen. The homogenate was centrifuged at $10,000 \mathrm{~g}$ and $4{ }^{\circ} \mathrm{C}$ for $10 \mathrm{~min}$, and $50 \mu \mathrm{L}$ of the resultant supernatant was diluted with distilled water and added to $250 \mu \mathrm{L}$ of the Folin-Ciocalteu reagent $(0.2 \mathrm{~mol} / \mathrm{L})$. The reaction mixture reacted for $5 \mathrm{~min}$ before $50 \mu \mathrm{L}$ of $20 \% \mathrm{Na}_{2} \mathrm{CO}_{3}$ was added. The samples were then incubated at room temperature in darkness for $60 \mathrm{~min}$, and the absorbance was measured at $760 \mathrm{~nm}$. A calibration curve was obtained using $0-4000 \mathrm{mg}$ of gallic acid per milliliter and used to calculate the total phenolic content of the tea seedling leaves. 


\section{Determination of antioxidant enzyme activity}

The fresh leaves $(0.2 \mathrm{~g})$ of tea seedling were used immediately for enzyme extraction after excised, and subsequently homogenized with liquid nitrogen. Sodium phosphate buffer (50 mM; pH 6.8) was used as extraction buffer. The mixture of tea leaves extraction was then centrifuged at 15,000 $\mathrm{g}$ for $30 \mathrm{~min}$, and the resultant supernatant was used in the following enzyme activity assays. CAT activity was analyzed as described in previous studies (Chao et al. 2012; Kato and Shimizu 1985). Decreasing in absorbance at $240 \mathrm{~nm}$ was observed using a spectrophotometer (Metertec SP8001) which implied the decrease of $\mathrm{H}_{2} \mathrm{O}_{2}$. Activity of CAT was calculated on the basis of the extinction coefficient $\left(40 \mathrm{mM}^{-1} \mathrm{~cm}^{-1}\right.$ at $240 \mathrm{~nm}$ ) of $\mathrm{H}_{2} \mathrm{O}_{2}$. One unit of CAT was defined as the enzyme amount that degraded $1 \mu \mathrm{mol}$ of $\mathrm{H}_{2} \mathrm{O}_{2}$ per minute. SOD activity assay was conducted based on Chao et al. (2012). The reaction buffer was Triethanolaminediethanolamine buffer (100 mM; pH 7.4) containing ethylenediaminetetraacetic acid/ $\mathrm{MnCl}_{2}(100 \mathrm{mM} / 50 \mathrm{mM}$, $\mathrm{pH}$ 7.4), $7.5 \mathrm{mM} \beta$-nicotinamide adenine dinucleotide $(\beta-\mathrm{NADH})$, and $10 \mathrm{mM}$ 2-mercaptoethanol and mixed with enzyme extract. The enzyme reaction was initiated by the addition of $\beta-\mathrm{NADH}$, and detected the absorbance at $340 \mathrm{~nm}$ for $10 \mathrm{~min}$. One unit of SOD was defined as the enzyme amount that inhibited the rate of $\beta$-NADH oxidation by $50 \%$. For APX activity assay, the decrease in the ascorbic acid (AsA) concentration was determined according to the decline in absorbance at $290 \mathrm{~nm}$, and the activity was calculated based on the extinction coefficient $\left(2.8 \mathrm{mM}^{-1} \mathrm{~cm}^{-1}\right.$ at $290 \mathrm{~nm}$ ) for AsA (Chao et al. 2012; Nakano and Asada 1981). Both soluble and ionically bound peroxidase (Total POX) activity assay was modified from previous studies (Lin and Kao 1999; MacAdam et al. 1992; Wu and Yang 2016). Samples were extracted by homogenized in liquid nitrogen with $50 \mathrm{mM}$ potassium phosphate buffer ( $\mathrm{pH} 5.8$ ) containing $0.8 \mathrm{M} \mathrm{KCl}$ buffer. The $50 \mathrm{mM}$ potassium phosphate buffer ( $\mathrm{pH}$ 5.8), $21.6 \mathrm{mM}$ guaiacol, and $39 \mathrm{mM} \mathrm{H} \mathrm{H}_{2}$ were added into enzyme extract for reaction. The enzyme reaction was initiated by adding $\mathrm{H}_{2} \mathrm{O}_{2}$, the absorbance was measured at $470 \mathrm{~nm}$ for $3 \mathrm{~min}$. Total POX activity was calculated on the basis of the extinction coefficient of $26.6 \mathrm{mM}^{-1} \mathrm{~cm}^{-1}$ at $470 \mathrm{~nm}$ for tetraguaiacol. One unit of POX was defined as the enzyme amount that caused the formation of $1 \mu \mathrm{mol}$ of tetraguaiacol per minute.

\section{Determination of DPPH radical scavenging activity}

Potential antioxidant activity was determined using DPPH (2,2-diphenyl-1-picrylhydrazyl) according to Tadolini et al. (2000) with some modifications. The sample $(0.1 \mathrm{~g})$ extract was added to $1.0 \mathrm{~mL}$ of $75 \%$ ethanol. The mixture was shaken for $120 \mathrm{~min}$ through vortexing and left to centrifuge at $10,000 \mathrm{~g}$ and room temperature in darkness for $10 \mathrm{~min}$. The absorbance for the sample was measured using a SpectraMax M2 spectrophotometer at $521 \mathrm{~nm}$ against an ethanol blank. A control sample $\left(\triangle \mathrm{A}_{\mathrm{DPPH}}\right)$ was extracted after adding $0.19 \mathrm{mM} \mathrm{DPPH}$ solution to $0.2 \mathrm{~mL}$ of the respective extraction solvent. Every sample was extracted in triplicate, and the results were calculated based on four biologically independent experiments. The percentage of DPPH free radicals scavenged in the sample was calculated using the following equation:

$$
\begin{aligned}
& \text { DPPH free radical scavenging ratio (\%) } \\
& \quad=\left[\left(\Delta \mathrm{A}_{\mathrm{DPPH}}-\Delta \mathrm{A}_{\text {sample }}+\mathrm{DPPH}\right) /\left(\Delta \mathrm{A}_{\mathrm{DPPH}}\right)\right] \times 100
\end{aligned}
$$

Oxygen radical absorbance capacity (ORAC) measurement The ORAC assay was by using a modified form of the method described by Ou et al. (2001). The ORAC assay was measured the capacity of antioxidative compounds in test materials to inhibit the decrease in fluorescence induced by the peroxyl radical $2,2^{\prime}$-azobis (2-amidinopropane) dihydrochloride (AAPH). The samples $(0.1 \mathrm{~g})$ were homogenized with liquid nitrogen and added to $1 \mathrm{~mL}$ of double distilled $\mathrm{H}_{2} \mathrm{O}$ for incubation $10 \mathrm{~min}$ at $100{ }^{\circ} \mathrm{C}$. The $20 \mu \mathrm{L}$ extract was added to $120 \mu \mathrm{L} 120 \mathrm{nM}$ disodium fluorescein solution (72 nM, final concentration) and preincubated for $15 \mathrm{~min}$ at $37{ }^{\circ} \mathrm{C}$. The mixture was rapidly added to $60 \mu \mathrm{L}$ of $40 \mathrm{mM}$ AAPH in the well of the microplate and the microplate was immediately placed in the reader, and the fluorescence was recorded at an $485 \mathrm{~nm}$ (excitation wavelength) and $535 \mathrm{~nm}$ (emission wavelength) every $1 \mathrm{~min}$ for $60 \mathrm{~min}$. The blank (FL + AAPH) with phosphate buffer instead of the antioxidant solution and Trolox was used as the standard. All the reaction mixtures were detected in duplicate, and at least three independent assays were performed for each sample. Fluorescence was measured and recorded every $1 \mathrm{~min}(f 0, f 1$, $f 2, f 3, \ldots, f 60)$ until the fluorescence began to decline. The curves of antioxidant were initially normalized to the blank curve corresponding to the same assay by the factor fluorescence ${ }_{\text {blank }, t=0} /$ fluorescence $_{\text {sample }, t=0}$. Based on the normalized curves, the area under the fluorescence decay curve (AUC) was calculated as follows:

$$
\begin{aligned}
\mathrm{AUC}=1+ & \sum_{i=1}^{i=60} f i / f 0 \\
\mathrm{ORAC}_{\mathrm{FL}}= & \frac{\mathrm{AUC}_{\text {sample }}-\mathrm{AUC}_{\text {blank }}}{\mathrm{AUC}_{\text {trolox }}-\mathrm{AUC}_{\text {blank }}} \\
& \times \text { Trolox molarity } \times \text { sample dilution. }
\end{aligned}
$$

Each sample was calculated by subtracting the AUC corresponding to the blank. Equations for regression 
between the net AUC and antioxidant concentration were calculated for all samples. The concentrations are expressed as micrograms of Trolox equivalents per $100 \mathrm{mg}$ of fresh weight.

\section{Results}

\section{Content and degree of catechin galloylation were higher} in response to ethylene signaling

The level of catechin galloylation was determined through HPLC after 5 days of treatment with the ethylene precursor $A C C$, and the content of total catechin and values of $\mathrm{ECG} /(\mathrm{EC}+\mathrm{ECG})$ and EGCG/(EGC+EGCG) were calculated. The HPLC profiles revealed the corresponding peaks to caffeine and the four major catechins including EC, ECG, EGC, and EGCG (Fig. 1a). The total catechin content was higher in the tea seedlings treated with ACC than in the control sample (Fig. 1b). The degrees of EGC and EC galloylation were higher after ACC treatment in comparison with the control sample (Fig. 1c).

\section{Content of anthocyanins, flavonoids, and total polyphenol was enhanced after the ACC treatment}

To investigate the effects of ethylene signaling in the secondary metabolic pathway, the contents of anthocyanins, flavonoids, and total polyphenol were determined after ACC treatment in tea seedlings. The contents of anthocyanins (Fig. 2a), flavonoids (Fig. 2b), and total polyphenols (Fig. 2c) were higher in the tea seedlings that underwent ACC treatment than in the control sample. These findings show that ethylene signaling was involved in the
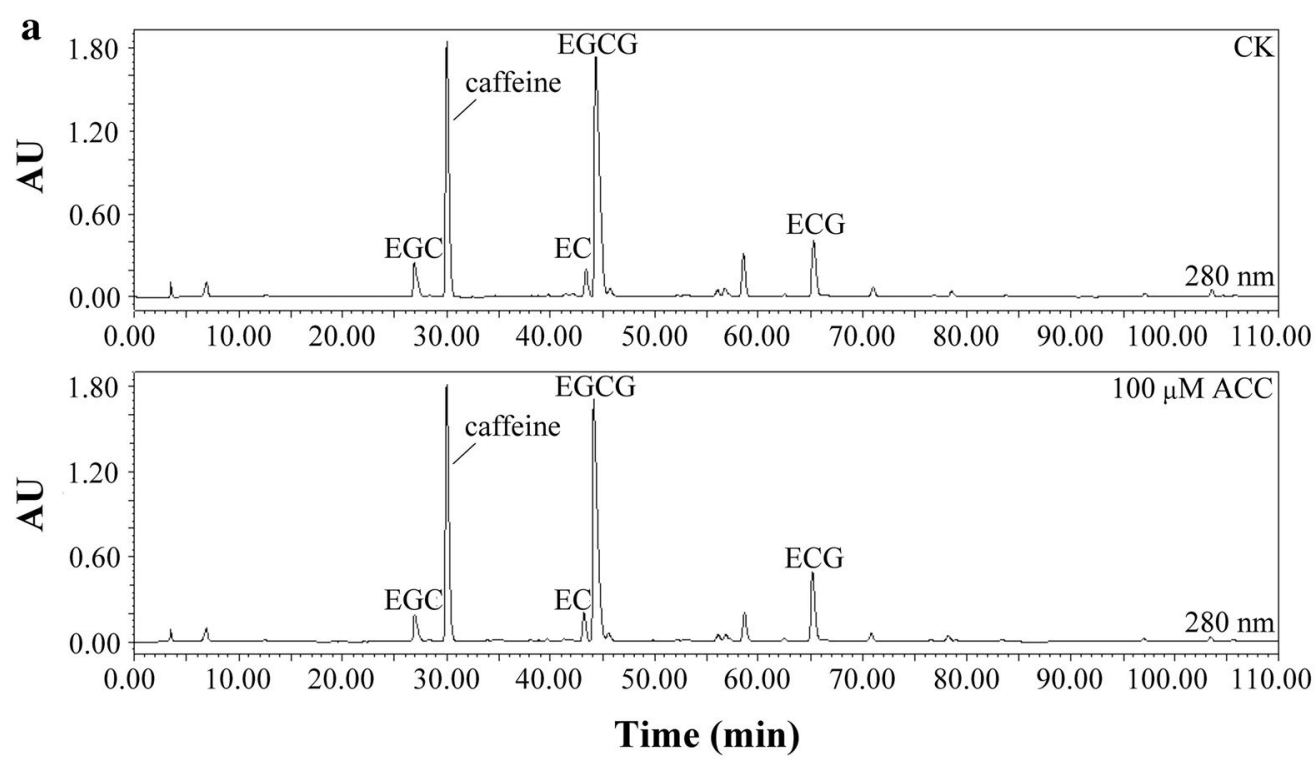

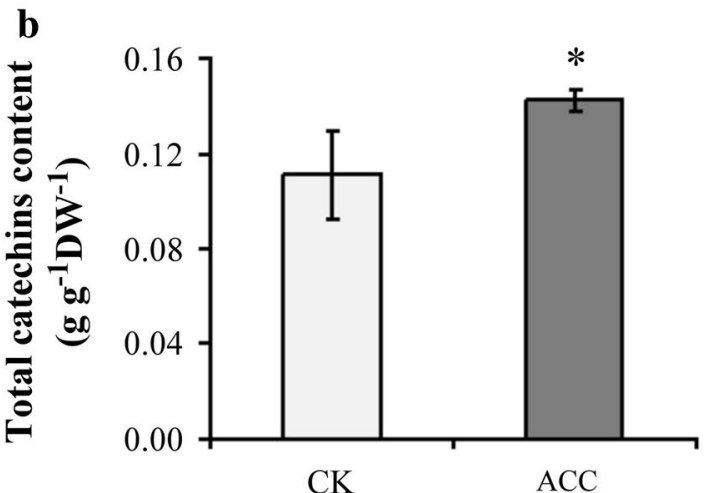

Treatment

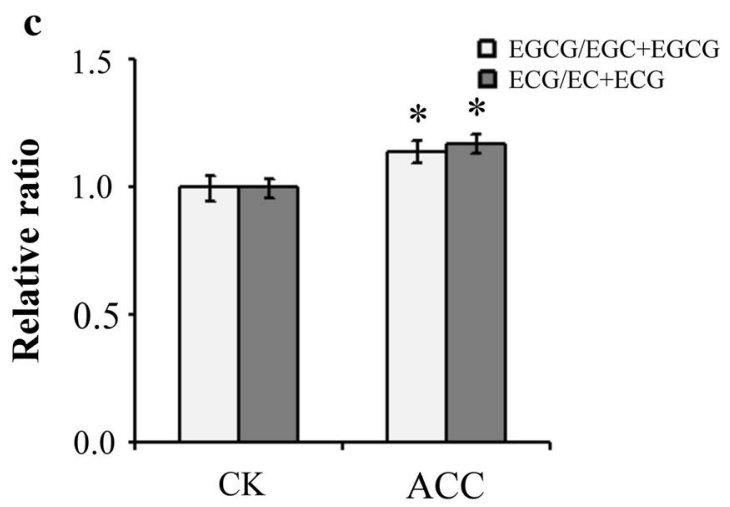

Treatment

Fig. 1 Effects of ethylene signaling on the content and degree of catechin galloylation in tea seedlings. a Caffeine and the four major catechins, EC, EGC, ECG, and EGCG, were detected. b Total catechin content. $\mathbf{c}$ The relative ratio was calculated as follows: ECG/(EC+ECG) and EGCG/ $(E G C+E G C G)$. Data points are mean \pm SD of five biologically independent experiments. Asterisks indicate significant differences from the control sample $(P<0.05 ;$ Student's $t$ test) 


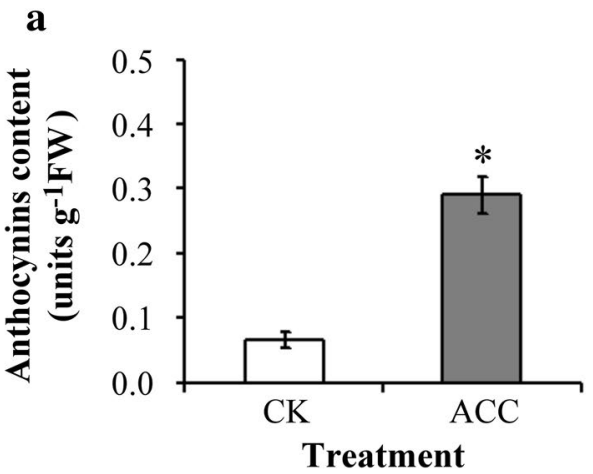

b

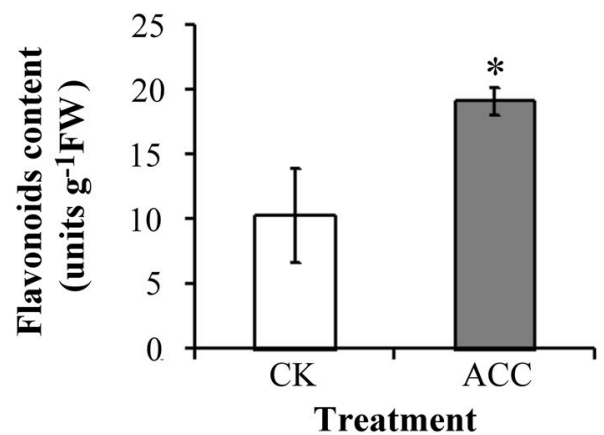

c
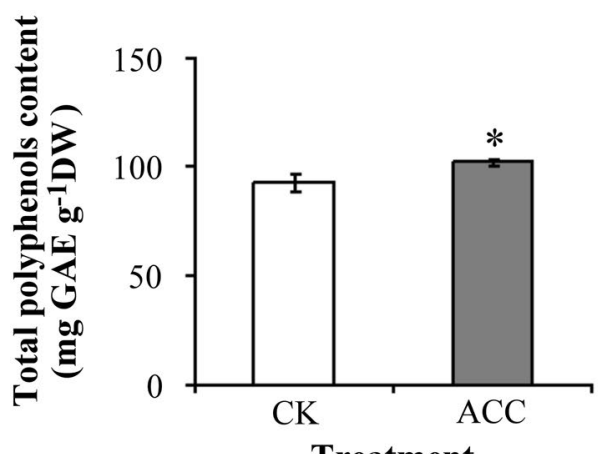

Treatment

Fig. 2 Effects of ethylene signaling on the content of secondary metabolites in tea seedlings. The content of $\mathbf{a}$ anthocyanins, $\mathbf{b}$ flavonoids, and $\mathbf{c}$ total phenol was determined. Data points are mean \pm SD of three biologically independent experiments. Asterisks indicate significant differences from the control sample $(P<0.05$; Student's $t$ test $)$

modulation of the metabolic pathway in the oolong tea seedlings.

\section{Effects of ethylene signaling on antioxidant enzyme activity}

To evaluate the cellular free radical scavenging capacity of the antioxidant enzymes in oolong tea seedlings after ACC treatment, the activity of antioxidant enzymes such as SOD, CAT, APX, and total POX was evaluated.
Our results show that the activity of SOD (Fig. 3a), CAT (Fig. 3b), and POX (Fig. 3d) decreased under ACC treatment for 5 days. By contrast, APX activity significantly increased under ACC treatment for 5 days (Fig. 3c).

\section{Antioxidant capacity was increased on modulation of ethylene signaling}

To evaluate the effects of ethylene signaling on the antioxidant capacity of tea seedlings, assays of DPPH and ORAC were conducted. After ACC treatment for 5 days, $64 \%$ of the DPPH radicals were scavenged, whereas only $57 \%$ were scavenged in the control sample (Fig. 4). The ORAC-FL assay indicated that the ORAC was enhanced by ACC treatment (Fig. 5).

\section{Discussion}

Ethylene signaling involved in the flavonoid synthesis pathway in tea seedlings

Ethylene is a vital phytohormone in plants. Some previous studies have demonstrated that ethylene signaling is involved in metabolic pathways; for example, ethylene treatment increased total polyphenol and anthocyanin after grape harvesting (Bellincontro et al. 2006). Studies have indicated that exogenous application of $\alpha$-aminoisobutyric acid as an analogue of ACC maintained higher catechin content in longan fruit pericarp during storage (Wang et al. 2015). Catechins are the most vital secondary metabolites in tea plants which already demonstrated galloylated catechins presented stronger positive health benefit potential than that of nongalloylated catechins (Chen et al. 2014). The results of this study show increases in not only the total catechin content but also the level of EGC and EC galloylation after ACC treatment in seedlings of oolong tea (Fig. 1). Moreover, the content of anthocyanins, flavonoids, and total polyphenol was enhanced by ACC treatment in oolong tea seedlings (Fig. 2). Our results indicate that ethylene signaling was involved in the flavonoid biosynthesis pathway and modulated the catechin galloylation level in oolong tea seedlings.

\section{Role of ethylene signaling in antioxidant enzyme activity and antioxidant capacity in oolong tea seedlings}

Reactive oxygen species (ROSs) are produced in chloroplasts, mitochondria, and peroxisomes as a typical product of plant cellular metabolism. Several studies have reported that plants exhibited excessive ROS production when they were subjected to biotic or abiotic stress, and the consequent oxidant-antioxidant imbalance activated factors responsible for cell injury (Liu et al. 2013; Wang et al. 2012; Wu and Yang 2016). Antioxidants are crucial because their main responsibility is the scavenging 

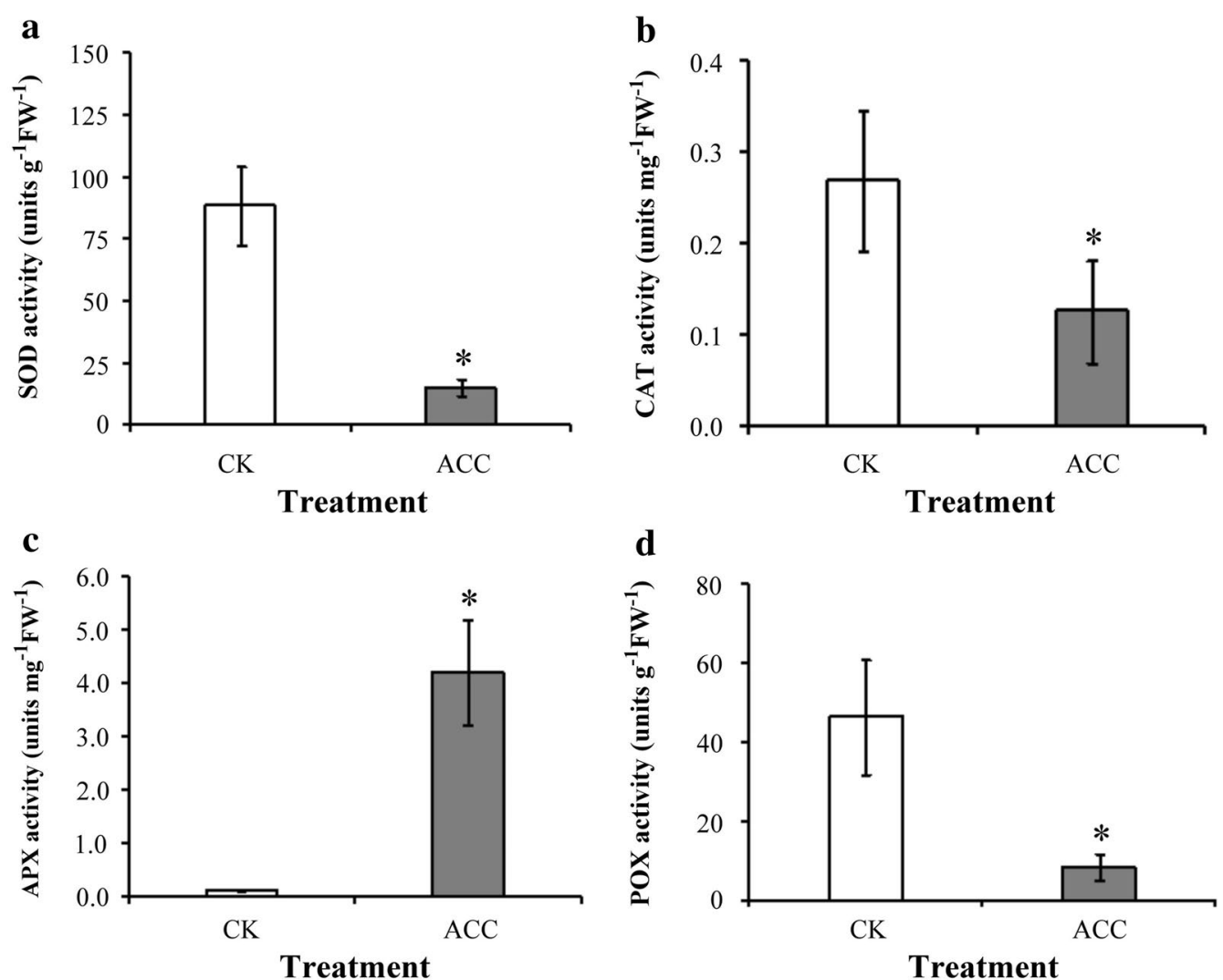

Fig. 3 CAT, APX, SOD, and POX activity in tea seedlings on modulation of ethylene signaling. Enzyme activity was detected from the detached tea seedling samples with one tip and two leaves after treatment with the control (CK) or $100 \mu \mathrm{M}$ ACC for 5 days.CAT, APX, SOD, and total POX activity was determined. Data points are mean \pm SD of five biologically independent experiments. Asterisks indicate significant differences from the control sample $(P<0.05 ;$ Student's $t$ test $)$

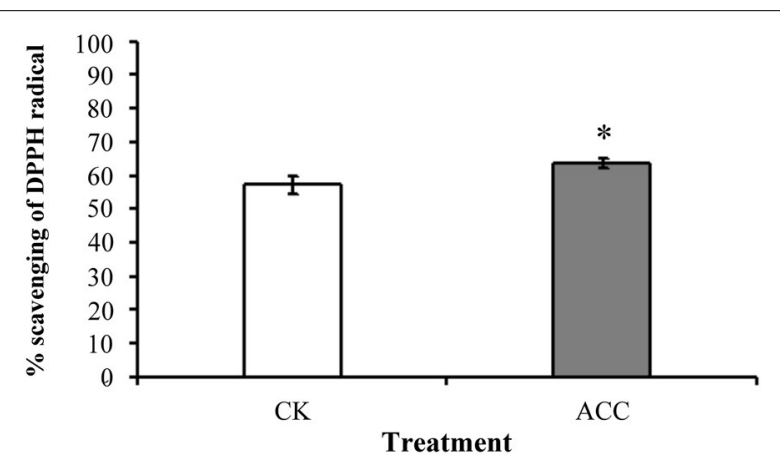

Fig. 4 DPPH radical scavenging activity in tea seedlings on modulation of ethylene signaling. The scavenging ratios of DPPH radicals were determined from the detached tea seedling samples after treatment with the control (CK) or $100 \mu \mathrm{M}$ ACC for 5 days. Data points are mean \pm SD of three biologically independent experiments. Asterisks indicate significant differences from the control sample $(P<0.05$; Student's $t$ test)

of ROSs, which is indispensable in protecting cell constituents from damage. Studies on phenolic compounds have shown that they are involved in plant antioxidant defense systems (Posmyk et al. 2009). Ethylene is known involved in the abscission of vegetative and reproductive organs and senescence under stress conditions. Ethylene reduced chilling damage during fruit storage and increased the activity of APX enzymes (Lafuente et al. 2004). Our data showed that using the ethylene precursor ACC to treat tea seedlings promoted the induction of phenolic compounds (Fig. 2). Therefore, ethylene signaling presumably affects the tea plant antioxidant system balance. The antioxidant enzyme activity assay revealed that the SOD, CAT, and POX activity in tea seedlings (Fig. 3a, b, d, respectively) was decreased after ACC treatment. By contrast, the APX activity was significantly increased after ACC treatment (Fig. 3c). These findings suggest that ethylene signaling promoted higher phenolic compound accumulation, which has implications for antioxidant enzyme activity in tea seedlings. The antioxidant capacity was determined by evaluating ORAC and DPPH free radical scavenging (Figs. 4 and 5). Our results indicate that higher antioxidant capacity in oolong tea seedlings can be achieved through ethylene signaling. 


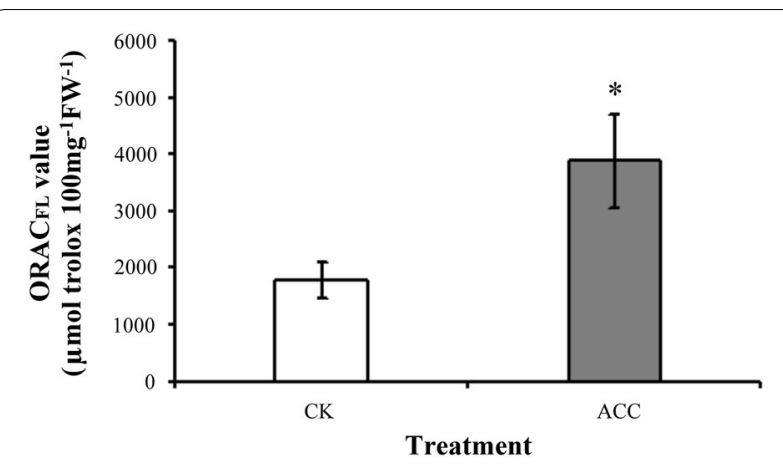

Fig. 5 ORAC in tea seedlings on modulation of ethylene signaling. ORAC-FL values were determined from the detached tea seedling samples after treatment with the control (CK) or $100 \mu \mathrm{M}$ ACC for 5 days. Data points are mean \pm SD of three biologically independent experiments. Asterisks indicate significant differences from the control sample ( $P<0.05$; Student's $t$ test)

\section{Conclusions}

According to our results suggest that the ethylene signaling involved in the secondary metabolic pathway induced the accumulation of phenolic compounds and catechin galloylation and reduced antioxidant enzyme activity in the tea seedlings. The antioxidant capacity was positively correlated with phenolic compound accumulation during ethylene signaling. Whether or not the ACC signal is possible involved to regulate plant secondary metabolic pathway requires further research.

\begin{abstract}
Abbreviations
ACC: 1-aminocyclopropane-1-carboxylic acid; EC: epicatechin; ECG: epicatechin-gallate; EGC: epigallocatechin; EGCG: epigallocatechin-gallate; HPLC: high-performance liquid chromatography; CAT: catalase; SOD: superoxide dismutase; POX: total peroxidase; APX: ascorbate peroxidase; ORAC: oxygen radical absorption capacity; DPPH: 2,2-diphenyl-1-picrylhydrazyl; ROS: reactive oxygen species.
\end{abstract}

\section{Authors' contributions}

The work presented here was carried out in collaboration between all authors. SWK, GHC and CTC carried out the experiments and analyzed the data. Dr. JTCT helps the analysis of HPLC data. Dr. CYY defined the research theme, designed experiments and wrote the manuscript. All authors read and approved the final manuscript.

\section{Author details}

1 Department of Agronomy, National Chung Hsing University, Taichung 40227, Taiwan. ${ }^{2}$ Graduate Institute of Biotechnology, National Chung Hsing University, Taichung 40227, Taiwan.

\section{Acknowledgements}

Not applicable.

\section{Competing interests}

The authors declare that they have no competing interests.

\section{Availability of data and materials}

Not applicable.

\section{Consent for publication}

Authors agree to the terms of the Springer Open Copyright and License Agreement.

\section{Ethics approval and consent to participate}

Not applicable.

\section{Funding}

This study was funded by the National Science Council, Taiwan, R.O.C. (NSC 104-2311-B-005-006).

\section{Publisher's Note}

Springer Nature remains neutral with regard to jurisdictional claims in published maps and institutional affiliations.

Received: 10 November 2017 Accepted: 29 March 2018

Published online: 03 April 2018

\section{References}

Abeynayake SW, Panter S, Chapman R, Webster T, Rochfort S, Mouradov A, Spangenberg G (2012) Biosynthesis of proanthocyanidins in white clover flowers: cross talk within the flavonoid pathway. Plant Physiol 158(2):666-678

Bellincontro A, Fardelli A, De Santis D, Botondi R, Mencarelli F (2006) Postharvest ethylene and 1-MCP treatments both affect phenols, anthocyanins, and aromatic quality of Aleatico grapes and wine. Aust J Grape Wine R 12(2):141-149

Boller T, Herner RC, Kende H (1979) Assay for and enzymatic formation of an ethylene precursor, 1-aminocyclopropane-1-carboxylic acid. Planta 145(3):293-303

Bosch M, Berger S, Schaller A, Stintzi A (2014) Jasmonate-dependent induction of polyphenol oxidase activity in tomato foliage is important for defense against Spodoptera exigua but not against Manduca sexta. BMC Plant Biol 14:257

Chao YY, Chou TS, Kao CH (2012) Involvement of abscisic acid and hydrogen peroxide in regulating the activities of antioxidant enzymes in leaves of rice seedlings under magnesium deficiency. Plant Growth Regul 66:1-8

Chen GH, Yang CY, Lee SJ, Wu CC, Tzen JTC (2014) Catechin content and the degree of its galloylation in oolong tea are inversely correlated with cultivation altitude. J Food Drug Anal 22(3):303-309

Kato M, Shimizu S (1985) Chlorophyll metabolism in higher plants VI. involvement of peroxidase in chlorophyll degradation. Plant Cell Physiol 26(7):1291-1301

Lafuente MT, Sala JM, Zacarias L (2004) Active oxygen detoxifying enzymes and phenylalanine ammonia-lyase in the ethylene-induced chilling tolerance in citrus fruit. J Agric Food Chem 52(11):3606-3611

Lee TM, Chu C (1992) Ethylene-induced polyamine accumulation in rice (Oryza sativa L.) coleoptiles. Plant Physiol 100(1):238-245

Lewis DR, Ramirez MV, Miller ND, Vallabhaneni P, Ray WK, Helm RF, Winkel BSJ, Muday GK (2011) Auxin and ethylene induce flavonol accumulation through distinct transcriptional networks. Plant Physiol 156(1):144-164

Lin CC, Kao CH (1999) NaCl induced changes in ionically bound peroxidase activity in roots of rice seedlings. Plant Soil 216:147-153

Liu J, Wu YC, Kan J, Wang Y, Jin CH (2013) Changes in reactive oxygen species production and antioxidant enzyme activity of Agaricus bisporus harvested at different stages of maturity. J Sci Food Agric 93(9):2201-2206

MacAdam JW, Nelson CJ, Sharp RE (1992) Peroxidase activity in the leaf elongation zone of tall fescue. Plant Physiol 99(3):872-878

Nakano Y, Asada K (1981) Hydrogen peroxide is scavenged by ascorbate-specific peroxidase in spinach chloroplasts. Plant Cell Physiol 22(5):867-880

Ou BX, Hampsch-Woodill M, Prior RL (2001) Development and validation of an improved oxygen radical absorbance capacity assay using fluorescein as the fluorescent probe. J Agric Food Chem 49(10):4619-4626

Perva-Uzunalic A, Skerget M, Knez Z, Weinreich B, Otto F, Gruner S (2006) Extraction of active ingredients from green tea (Camellia sinensis): extraction efficiency of major catechins and caffeine. Food Chem 96(4):597-605 
Posmyk MM, Kontek R, Janas KM (2009) Antioxidant enzymes activity and phenolic compounds content in red cabbage seedlings exposed to copper stress. Ecotoxicol Environ Saf 72(2):596-602

Schaller GE (2012) Ethylene and the regulation of plant development. BMC Biol 10:9

Singleton VL, Rossi JA (1965) Colorimetry of total phenolics with phosphomolybdic-phosphotungstic acid reagents. Am J Enol Vitic 16:144-158

Sun BM, Zhu ZS, Cao PR, Chen H, Chen CM, Zhou X, Mao YH, Lei JJ, Jiang YP, Meng W, Wang YX, Liu SQ (2016) Purple foliage coloration in tea (Camellia sinensis L.) arises from activation of the R2R3-MYB transcription factor CsAN1. Sci Rep 6:32534. https://doi.org/10.1038/srep32534

Tadolini B, Juliano C, Piu L, Franconi F, Cabrini L (2000) Resveratrol inhibition of lipid peroxidation. Free Radic Res 33(1):105-114

Thipyapong P, Steffens JC (1997) Tomato polyphenol oxidase-differential response of the polyphenol oxidase $\mathrm{F}$ promoter to injuries and wound signals. Plant Physiol 115(2):409-418
Wang HB, Zhang ZQ, Xu LY, Huang XM, Pang XQ (2012) The effect of delay between heat treatment and cold storage on alleviation of chilling injury in banana fruit. J Sci Food Agric 92(13):2624-2629

Wang H, Zhi W, Qu HX, Lin HT, Jiang YM (2015) Application of alpha-aminoisobutyric acid and beta-aminoisobutyric acid inhibits pericarp browning of harvested longan fruit. Chem Cent J. https://doi.org/10.1186/ s13065-015-0124-1

Woolf AB, Plummer JA, Clemens J (1999) Maturity and temperature influence ethylene-promoted organ abscission in Camellia. N Z J Crop Hortic 27(1):33-41

Wu YS, Yang CY (2016) Physiological responses and expression profile of NADPH oxidase in rice (Oryza Sativa) seedlings under different levels of submergence. Rice 9(1):2

Zhou QQ, Sun WJ, Lai ZX (2016) Differential expression of genes in purpleshoot tea tender leaves and mature leaves during leaf growth. J Sci Food Agric 96(6):1982-1989

\section{Submit your manuscript to a SpringerOpen ${ }^{\circ}$ journal and benefit from:}

- Convenient online submission

- Rigorous peer review

- Open access: articles freely available online

- High visibility within the field

- Retaining the copyright to your article

Submit your next manuscript at springeropen.com 